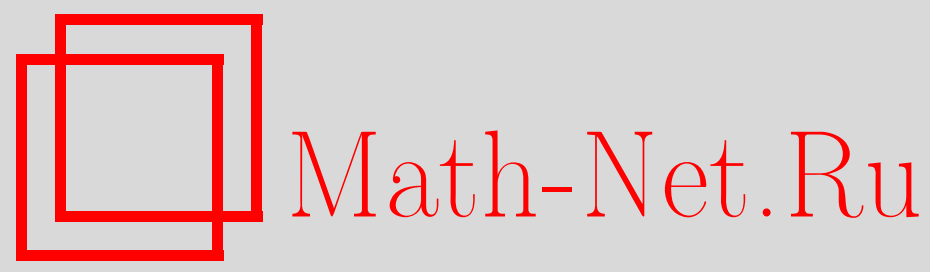

О. В. Лычагина, Вырожденные пуассоновы структуры в размерности 3, Матем. заметки, 1998, том 63, выпуск $4,579-592$

DOI: https://doi.org/10.4213/mzm1318

Использование Общероссийского математического портала Math-Net.Ru подразумевает, что вы прочитали и согласны с пользовательским соглашением http://www . mathnet.ru/rus/agreement

Параметры загрузки:

IP : 54.198 .187 .58

26 апреля 2023 г., 02:43:56

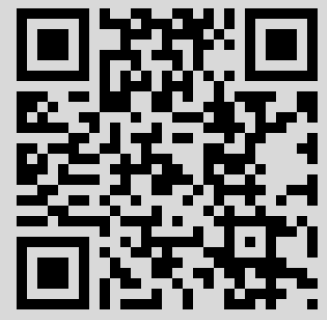


УДК 514.763 .85

\section{ВЫРОЖДЕННЫЕ ПУАССОНОВЫ СТРУКТУРЫ \\ В РАЗМЕРНОСТИ 3 \\ О.В. Лычагина}

В работе приведены формальные нормальные формы вырожденных пуассоновых структур в размерности 3. Исследование пуассоновых структур проведено с использованием спектральной последовательнсти на основе метода, введенного ранее. Этот метод, в частности, позволяет другим способом получить известные результаты о линеаризации пуассоновых структур с полупростой линейной частью. Однако, в размерности 3 имеются и нелинеаризуемые пуассоновы структуры.

Библиографияя: 9 названий.

Теорема о "расщеплении" Вейнстейна [1] сводит локальное изучение пуассоновых структур к изучению структур, обращающихся в 0 в фиксированной точке.

Проблема классификации таких структур изучалась многими математиками. В частности, Вейнстейн доказал формальную линеаризуемость вырожденной пуассоновой структуры с линейной частью, задающей полупростую алгебру Ли [1]. Кон доказал тот же результат в гладкой категории при дополнительном условии компактности типа для алгебры Ли [2]. Дюфур изучал гладкую линеаризуемость вырожденной пуассоновой структуры в трехмерном случае [3].

В работах [4], [5] найдены формальные нормальные формы вырожденных пуассоновых структур для случая произвольной размерности, описание которых приводится в терминах спектральной последовательности, сходящейся к формальньп пуассоновьм когомологиям. Более того, показано, что препятствия к линеаризации такой пуассоновой структуры лежат в группе вторых когомологий алгебры Ли $\mathscr{G}$, определяемой линейной частью вырожденной пуассоновой структуры.

В этой работе мы рассматриваем вырожденные пуассоновы структуры в размерности 3 с нетривиальной линейной частью и вычисляем соответствующие члены спектральной последовательности. Это дает описание (формальных) нормальных форм вырожденных пуассоновых структур на трехмерных многообразиях.

\section{§1. Введение}

Кратко напомним основные результаты, изложенные в работах [4], [5].

Пусть $\nabla$-пуассонова структура на гладком многообразии $M^{n}$, обращающаяся в 0 в точке $m \in M^{n}, \nabla_{m}=0$.

Рассмотрим комплекс для пуассоновых когомологий, определяемьй $\nabla$ :

$$
0 \longrightarrow \mathscr{D}_{0}=A \stackrel{\partial_{\nabla}}{\longrightarrow} \mathscr{D}_{1} \stackrel{\partial_{\nabla}}{\longrightarrow} \cdots \stackrel{\partial_{\nabla}}{\longrightarrow} \mathscr{D}_{i} \stackrel{\partial_{\nabla}}{\longrightarrow} \mathscr{D}_{i+1} \stackrel{\partial_{\nabla}}{\longrightarrow} \cdots \stackrel{\partial_{\nabla}}{\longrightarrow} \mathscr{D}_{n} \longrightarrow 0 .
$$

Здесь $\partial_{\nabla}(X):=(-1)^{i}[X, \nabla]$ для $\left.X \in \mathscr{D}_{i}, \llbracket \cdot \cdot, \cdot\right]-$ скобка Схоутена на алгебре поливекторных полей $\mathscr{D}_{*}=\sum_{i=0}^{\infty} \mathscr{D}_{i}$, где $\mathscr{D}_{i}-$ модули $i$-векторных полей на $M^{n}$. 
Обозначим через $\mu \subset A$ максимальный идеал, отвечающий точке $m: \mu=\{f \in$ $\left.C^{\infty}\left(M^{n}\right) \mid f(m)=0\right\}$, а через $\mu^{k}, k \geqslant 1$, идеал функций порядка малости $k$ в точке $m$. Соответственно через $\mu^{k} \mathscr{D}_{i}$ обозначим подмодуль $i$-векторных полей $\mathscr{D}_{i}$, имеющих в точке $m$ порядок малости $k$.

Через $[\sigma]_{p+1}$ обозначим $(p+1)$-джет в точке $m$ бивекторного поля $\sigma \in \mu^{p+1} \mathscr{D}_{2}$, т.е. класс $\sigma \bmod \mu^{p+2} \mathscr{D}_{2}$. Тогда линейная часть $[\nabla]_{1}$ определяет структуру алгебры Ли на кокасательном пространстве $T_{m}^{*} M^{n}$. Обозначим эту алгебру через $\mathscr{G}$.

Введем фильтрацию в комплекс (1), полагая $F_{p, q}=\mu^{p} \mathscr{D}_{p+q}$. Из условия $\nabla \in \mu \mathscr{D}_{2}$ следует, что $\partial_{\nabla}\left(\mu^{p} \mathscr{D}_{j}\right) \subset \mu^{p} \mathscr{D}_{j+1}$. Тем самым, дифференциал $\partial_{\nabla}$ сохраняет фильтрацию:

$$
\partial_{\nabla}\left(F_{p, q}\right) \subset F_{p, q+1} .
$$

Фильтрация $F_{p, q}$ определяет спектральную последовательность $\left(E_{r}^{p, q}, d_{r}\right)$, в которой

1) $E_{0}^{p, q}=S^{p} T_{m}^{*} \otimes_{\mathbb{R}} \Lambda^{p+q} T_{m}$;

2) $E_{1}^{p, q}=H^{p+q}\left(\mathscr{G}, S^{p} \mathscr{G}\right)$;

$3)$ для каждой пары чисел $(p, q)$ найдется число $r_{0}=r_{0}(p, q)$ такое, что

$$
E_{r_{0}}^{p, q}=E_{r_{0}+1}^{p, q}=\cdots=E_{\infty}^{p, q},
$$

где $d_{r}=0, r \geqslant r_{0}$ (т.е. спектральная последовательность стабилизируется).

Члены приведенной спектральной последовательности описывают нормальную форму $p$-го приближения следующим образом.

Теорема А [5]. Пусть бивекторные поля $v_{1}, \ldots, v_{r}, v_{i} \in \mu^{p} \mathscr{D}_{2}, p \geqslant 2$, таковы, что их образы порохсдают $E_{p-s+1}^{p, 2-p}$, где $2 \leqslant s \leqslant p+1$.

Тогда для каждой пуассоновой структуры вида $\nabla+\varepsilon$, где $\varepsilon \in \mu^{p} \mathscr{D}_{2}, p \geqslant 2$, существует локальный диффеоморфизм $\varphi$ такой, что $\varphi^{*}=\operatorname{id} \bmod \mu^{p} u$

$$
\varphi_{*}^{(2)}\left(\nabla-\sum_{i=1}^{r} c_{i} v_{i}\right)=(\nabla+\varepsilon) \bmod F_{p+1,1-p},
$$

әде $c_{i} \in \mathbb{R}, 1 \leqslant i \leqslant r$.

Введем следующее

ОПРЕДЕЛЕНИЕ. Класс когомологий $h \in H^{2}\left(\mathscr{G}, S^{p+1} \mathscr{G}\right)$ назовем допycmuмbым omносительно $\nabla^{\prime}$, если существует бивекторное поле $\sigma \in \mu^{p+1} \mathscr{D}_{2}$ такое, что класс когомологий $[\sigma]_{p+1}$ совпадает с $h$, a $\nabla^{\prime}+\sigma$ является (формальной) пуассоновой структурой.

Фиксируем пуассонову структуру $\nabla$ и определим по ней дерево пуассоновых структур следующим образом.

Вершина - линейная пуассонова структура $\nabla^{1}=[\nabla]_{1}$.

Уровень 1 - пуассоновы структуры вида $\nabla^{1}+\sigma_{2}$, где $\sigma_{2} \in \mu^{2} \mathscr{D}_{2}$, а класс $\left[\sigma_{2}\right]_{2}$ в $H^{2}\left(\mathscr{G}, S^{2} \mathscr{G}\right)$ пробегает все $\nabla^{1}$-допустимые структуры. Для каждого $\nabla^{1}$-допустимого класса $\lambda_{1} \in H^{2}\left(\mathscr{G}, S^{2} \mathscr{G}\right)$ зафиксируем одну из таких структур и обозначим ее через $\nabla_{\lambda_{1}}^{2}$.

Уровень 2 - пуассоновы структуры вида $\nabla_{\lambda_{1}}^{2}+\sigma_{3}$, где $\sigma_{3} \in \mu^{3} \mathscr{D}_{2}-$ представитель $\nabla_{\lambda_{1}}^{2}$-допустимых классов когомологий $\lambda_{2}$ в $E_{4-s(3)}^{3,-1}$. Здесь $E_{4-s(3)}^{3,-1}$ - член спектральной последовательности, построенной по пуассоновой структуре $\nabla_{\lambda_{1}}^{2}$. Обозначим эти структуры через $\nabla_{\lambda_{1}, \lambda_{2}}^{3}$.

Уровень $p$ - пуассоновы структуры вида $\nabla_{\lambda_{1}, \ldots, \lambda_{p-1}}^{p}+\sigma_{p+1}$, где $\sigma_{p+1} \in \mu^{p+1} \mathscr{D}_{2}-$ представитель $\nabla_{\lambda_{1}, \ldots, \lambda_{p-1}}^{p}$-допустимых классов когомологий $\lambda_{p}$ в $E_{p-s(p+1)+2}^{p+1,1-p}$. Здесь 
$E_{p-s(p+1)+2}^{p+1,1-p}-$ член спектральной последовательности, построенной по пуассоновой структуре $\nabla_{\lambda_{1}, \ldots, \lambda_{p-1}}^{p}$. Обозначим эти структуры через $\nabla_{\lambda_{1}, \ldots, \lambda_{p}}^{p+1}$.

Введем понятие ветви дерева (формальных) пуассоновых структур. Рассмотрим возникающие в дереве последовательности пуассоновых структур вида

$$
\nabla^{1}, \nabla_{\lambda_{1}}^{2}, \nabla_{\lambda_{1}, \lambda_{2}}^{3}, \ldots, \nabla_{\lambda_{1}, \ldots, \lambda_{p-1}}^{p}, \ldots
$$

Каждую такую последовательность назовем ветвью дерева. Для каждой ветви существует (формальная) пуассонова структура $\widetilde{\nabla}, p$-эквивалентная $p$-й точке ветвления ветви $\left((p-1)\right.$-му уровню ветви) - пуассоновой структуре $\nabla_{\lambda_{1}, \ldots, \lambda_{p-1}}^{p}$.

$\Phi$ иксируем структуру такого вида и обозначим ее через $\nabla_{\left\{\lambda_{i}\right\}_{1}^{\infty}}$. Будем отождествлять ветви и задающие их пуассоновы структуры.

В терминах построенного дерева пуассоновых структур мы получаем следующую теорему, описывающую нормальные формы вырожденных пуассоновых структур.

Теорема Б [5]. 1) Любая пуассонова структура вида $\nabla+\varepsilon$, дде $\varepsilon \in \mu^{2} \mathscr{D}_{2}$, формально әквивалентна одной из ветвей приведенного дерева, т.е. существу-

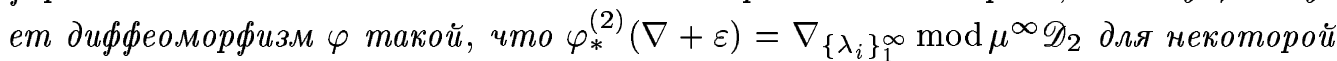
последовательности $\left\{\lambda_{i}\right\}_{1}^{\infty}, \varphi^{*}=\mathrm{id} \bmod \mu^{2} . B$ частности, $\nabla+\varepsilon$ әквивалентна $\bmod \mu^{p+1} \mathscr{D}_{2}$ пуассоновой структуре $\nabla_{\lambda_{1}, \ldots, \lambda_{p-1}}^{p}$ из $(p-1)$-го уровня дерева.

2) Две пуассоновы структуры, формально әквивалентные одной ветви дерева, формально әквивалентны.

Поскольку $E_{1}^{p, 2-p}=H^{2}\left(\mathscr{G}, S^{p} \mathscr{G}\right)$, мы получаем

СлЕДСТВИЕ 1. Достаточным условием линеаризачии вырожсденной пуассоновой структуры является равенство $H^{2}\left(\mathscr{G}, S^{p} \mathscr{G}\right)=0$.

Это дает линеаризуемость вырожденных пуассоновых структур с полупростой алгеброй $\mathscr{G}[1]$, а также линеаризуемость редуктивных алгебр Ли $\mathscr{G}$ с одномерным центром. Так, например, пуассоновы структуры линеаризуемы в случаях, ког да алгебра Ли $\mathscr{G}$ совпадает $\mathrm{c} \mathrm{gl}(n), \operatorname{csp}(n), \operatorname{cso}(n)$ (см. [4], [5]).

Здесь мы не рассматриваем случаи полупростых алгебр Ли $\mathscr{G}$ и абелевых алгебр $\mathscr{G}$ $\left([\nabla]_{1}=0\right)$. В первом случае пуассоновы структуры формально линеаризуемы [1], во втором - поиск нормальных форм требует развития методов вычисления когомологий алгебр Ли-Склянина [4]-[6].

Рассмотрим случай трехмерных пуассоновых многообразий. Трехмерные алгебры Ли $\mathscr{G}$, отличные от абелевых и полупростых, согласно классификации Бьянки [7] имеют следующий вид.

Обозначим коммутант алгебры $\mathscr{G}$ через $\mathscr{H}$. Возможны следующие три случая:

A) $\operatorname{dim} \mathscr{H}=1$, существует базис $x, y, z$ в $\mathscr{G}$ такой, что $[x, y]=x,[z, x]=0,[z, y]=0$;

B) $\operatorname{dim} \mathscr{H}=1$, сушествует базис $x, y, z$ в $\mathscr{G}$ такой, что $[x, y]=z,[z, x]=0,[z, y]=0$;

C) $\operatorname{dim} \mathscr{H}=2$, сушествует базис $x, y, z$ в $\mathscr{G}$ такой, что $[x, y]=0,[z, x]=a_{11} x+a_{12} y$, $[z, y]=a_{21} x+a_{22} y$, где матрица $\left(a_{i j}\right)$ невырождена.

Наша классификация (теоремы 1-4) вырожденных пуассоновых структур в размерности 3 излагается в соответствии с приведенной классификацией Бьянки.

\section{§2. Когомологии алгебр Ли}

\section{с коэффициентами в симметрической степени}

В этом параграфе мы приводим важнейший метод для вычисления первого члена 
спектральной последовательности $E_{1}^{p, q}$. В нашем случае $E_{1}^{p, q}=H^{p+q}(\mathscr{G}, A)$, где $A=$ $S^{*} \mathscr{G}=\sum_{i \geqslant 0} S^{i} \mathscr{G}$.

Рассмотрим комплекс

$$
0 \longrightarrow A \stackrel{d_{1}}{\longrightarrow} A \otimes \mathscr{G}^{*} \stackrel{d_{2}}{\longrightarrow} A \otimes \Lambda^{2} \mathscr{G}^{*} \longrightarrow \cdots
$$

с дифференциалом $d_{k}$, действующим по формуле $d_{k}(a \otimes \omega)=d a \wedge \omega+a \otimes d_{k} \omega$, где $d_{k} \omega-$ дифференциал для когомологий алгебр Ли с тривиальными коэффициентами, а $d a(x)=\widehat{x}(a)-$ действие элемента $x \in \mathscr{G}$ на алгебре $A$.

Мы будем использовать координатную запись. Пусть $x_{1}, \ldots, x_{n}$-базис в $\mathscr{G},\left[x_{i}, x_{j}\right]=$ $c_{i j}^{k} x_{k}$. Элемент $a \in S^{*} \mathscr{G}$, представимьй в виде $a=a_{\sigma_{1} \ldots \sigma_{n}} x_{1}^{\sigma_{1}} \circ \cdots \circ x_{n}^{\sigma_{n}}$, где "о" - симметрическое произведение, будем отождествлять с полиномом $a=a_{\sigma_{1} \ldots \sigma_{n}} x_{1}^{\sigma_{1}} \cdots x_{n}^{\sigma_{n}}$, где “." - произведение в полиномах, тем самым, рассматривая $S^{*} \mathscr{G}$ как алгебру однородных полиномов на $\mathscr{G}^{*}$.

Каждьй элемент $v \in \mathscr{G}$ действует присоединенным образом на $S^{*} \mathscr{G}$. А именно, $v$ определяет линейное векторное поле $\widehat{v}$ на $\mathscr{G}^{*}$, где $\widehat{v}=\sum_{i} v_{i}(x) \partial_{x_{i}}$, a $v_{i}=\widehat{v}\left(x_{i}\right)=\left[v, x_{i}\right]$. Тогда действие $\widehat{v}(a)$ совпадает с производной полинома $a$ вдоль векторного поля $\widehat{v}$.

Если $\operatorname{dim} \mathscr{G}=1$, то комплекс для вычисления когомологий алгебры Ли $\mathscr{G}$ с коэффициентами в $A$ имеет вид

$$
0 \longrightarrow A \stackrel{d}{\longrightarrow} A \otimes \mathscr{G}^{*} \longrightarrow 0
$$

где $d(a)(v)=\widehat{v}(a), v \in \mathscr{G}, a \in A$.

Поэтому $H^{i}(\mathscr{G}, A)=0$, если $i \geqslant 2$, и $H^{0}(\mathscr{G}, A)=A^{\mathscr{G}}=\{a \in A \mid v(a)=0$ для всех $v \in \mathscr{G}\}, H^{1}(\mathscr{G}, A)=A / \mathscr{G}(A)$.

Для вычисления групп когомологий $H^{i}\left(\mathscr{G}, S^{*} \mathscr{G}\right), i=0,1,2$, мы будем использовать спектральную последовательность Серра-Хохшильда $\left(E_{r}^{p, q}, d_{r}^{p, q}\right)$, построенную по паре $(\mathscr{G}, \mathscr{H})[8]$. Второй член этой последовательности имеет вид

$$
E_{2}^{p, q}=H^{p}\left(\mathscr{G} / \mathscr{H}, H^{q}(\mathscr{H}, A)\right) .
$$

Далее, во всех вычислениях, если аргумент функции не указан, предполагается, что функция зависит от всех аргументов.

\section{§ 3. Нормальные формы}

3.1. Пуассоновы структуры в размерности 2. В этом пунктемы проиллюстрируем наш метод для двумерных вырожденных пуассоновых структур с нетривиальной линейной частью. Теорема о линеаризуемости таких структур приведена, например, в работе [9].

Комплекс для вычисления когомологий двумерной алгебры $\mathscr{G}$ с базисом $x, y$ имеет вид

$$
0 \longrightarrow A \stackrel{d_{1}}{\longrightarrow} A \otimes \mathscr{G}^{*} \stackrel{d_{2}}{\longrightarrow} A \otimes \Lambda^{2} \mathscr{G}^{*} \longrightarrow 0,
$$

где $d_{1} p=\widehat{x}(p) \partial_{x}+\widehat{y}(p) \partial_{y}$ для $p \in A$ и

$$
d_{2}\left(p_{1} \partial_{x}+p_{2} \partial_{y}\right)=\left(\widehat{x}\left(p_{2}\right)-\widehat{y}\left(p_{1}\right)-\left(p_{1} \partial_{x}+p_{2} \partial_{y}\right)([x, y])\right) \partial_{x} \wedge \partial_{y} .
$$

Пусть $\mathscr{G}$ - разрешимая двумерная алгебра Ли. Выберем базис $x, y$ в $\mathscr{G}$ такой, что $[x, y]=x$. Пусть $\mathscr{H}$ - коммутант алгебры $\mathscr{G}, \mathscr{H} \subset \mathscr{G}$. Тогда $\mathscr{H}=\mathbb{R} \cdot x, \mathscr{G} / \mathscr{H}=\mathbb{R} \cdot y$ и $\widehat{x}=x \partial_{y}, \widehat{y}=-x \partial_{x}$.

Проведем вычисление груп когомологий $H^{i}\left(\mathscr{G}, S^{*} \mathscr{G}\right), i=0,1,2$, с помошью спектральной последовательности Серра-Хохшильда $\left(E_{r}^{p, q}, d_{r}^{p, q}\right)$, сходящейся к когомологиям алгебры $\mathscr{G}[8]$. 
Комплекс для вычисления когомологий $H^{*}(\mathscr{H}, A)$ имеет следующий вид:

$$
0 \longrightarrow A \longrightarrow A \otimes \mathscr{H}^{*} \longrightarrow 0 \text {. }
$$

Поэтому

$$
\begin{gathered}
H^{0}(\mathscr{H}, A)=\left\{p \in \mathbb{R}[x, y] \mid x \frac{\partial p}{\partial y}=0\right\}=\mathbb{R}[x], \\
H^{1}(\mathscr{H}, A)=\frac{\left\{p \partial_{x}\right\}}{\left\{x \cdot \partial q / \partial y \cdot \partial_{x}\right\}}=\left\{p(y) \partial_{x}\right\}=\mathbb{R}[y] \partial_{x} \simeq \mathbb{R}[y] .
\end{gathered}
$$

Для вычисления членов $E_{2}^{p, q}$ воспользуемся комплексами

$$
\begin{gathered}
0 \longrightarrow \mathbb{R}[x] \longrightarrow(\mathscr{G} / \mathscr{H})^{*} \otimes \mathbb{R}[x] \longrightarrow 0, \\
0 \longrightarrow \mathbb{R}[y] \partial_{x} \stackrel{\text { iso }}{\longrightarrow}(\mathscr{G} / \mathscr{H})^{*} \otimes\left(\mathbb{R}[y] \partial_{x}\right) \longrightarrow 0,
\end{gathered}
$$

где изоморфизм iso определяется следуюшим образом:

$$
\begin{aligned}
p(y) \partial_{x} \mapsto d\left(p(y) \partial_{x}\right) & =\widehat{y}\left(p(y) \partial_{x}\right) \wedge \partial_{y}=-x \partial_{x}\left(p(y) \partial_{x}\right) \wedge \partial_{y} \\
& =-p(y)\left[x \partial_{x}, \partial_{x}\right] \wedge \partial_{y}=-p(y) \partial_{x} \wedge \partial_{y}
\end{aligned}
$$

Из (2) следует, что

$$
E_{2}^{0,0}=H^{0}(\mathscr{G} / \mathscr{H}, \mathbb{R}[x])=(\mathbb{R}[x])^{\mathscr{G} / \mathscr{H}} \simeq \mathbb{R}, \quad E_{2}^{1,0} \simeq E_{2}^{0,0} \simeq \mathbb{R},
$$

а из (3)-

$$
E_{2}^{0,1} \simeq E_{2}^{1,1} \simeq H^{0}\left(\mathscr{G} / \mathscr{H}, \mathbb{R}[y] \partial_{x}\right)=\left(\mathbb{R}[y] \partial_{x}\right)^{\mathscr{G} / \mathscr{H}}=0 .
$$

Таким образом, член $E_{2}$ спектральной последовательности имеет вид

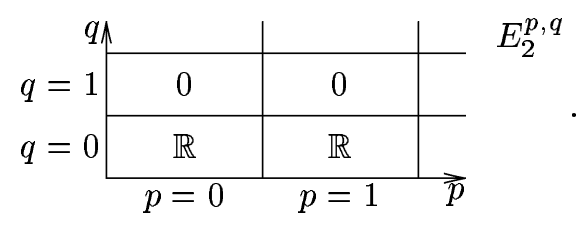

Из соображений размерности $d_{2}^{p, q}=d_{3}^{p, q}=\cdots=0$, тем самым, $E_{2}^{p, q}=E_{\infty}^{p, q}$. Следовательно, $H^{0}\left(\mathscr{G}, S^{r} \mathscr{G}\right)=H^{1}\left(\mathscr{G}, S^{r} \mathscr{G}\right)=\delta_{0}^{r} \cdot \mathbb{R}$, а $H^{2}(\mathscr{G}, A)=0$. Поэтому, используя теорему Б, получаем следующее

СлЕДСТвиЕ 2. Любая вырожденная двумерная пуассонова структура с нетривиальной линейной частью формально линеаризуема.

3.2. Алгебры типа А. В данном случае идеал $\mathscr{H} \subset \mathscr{G}$ порожден $x, \mathscr{G} / \mathscr{H}$ порожден $y, z$ и векторные поля $\widehat{x}, \widehat{y}, \widehat{z}$ имеют вид $\widehat{x}=x \partial_{y}, \widehat{y}=-x \partial_{x}, \widehat{z}=0$ соответственно.

Вычислим сначала $H^{q}(\mathscr{H}, A)$ для $q=0,1$. Заметим, что в комплексе

$$
0 \longrightarrow \mathbb{R}[x, y, z] \stackrel{d_{1}}{\longrightarrow} \mathbb{R}[x, y, z] \otimes \mathscr{H}^{*} \longrightarrow 0
$$

дифференциал действует следуюшим образом:

$$
d_{1}: p \mapsto d_{1} p=\widehat{x}(p) \partial_{x}=x \frac{\partial p}{\partial y} \partial_{x}
$$




\section{Поэтому}

$$
H^{0}(\mathscr{H}, A)=A^{\mathscr{H}}=\mathbb{R}[x, z], \quad H^{1}(\mathscr{H}, A)=\frac{\left\{p \otimes \partial_{x}\right\}}{\left\{x \cdot \partial q / \partial y \cdot \partial_{x}\right\}}=\mathbb{R}[y, z] \partial_{x} .
$$

Для вычисления когомологий $\mathscr{G} / \mathscr{H}$ рассмотрим комплекс

$$
0 \longrightarrow \mathbb{R}[x, z] \stackrel{d_{1}}{\longrightarrow} \mathbb{R}[x, z] \otimes(\mathscr{G} / \mathscr{H})^{*} \stackrel{d_{2}}{\longrightarrow} \mathbb{R}[x, z] \otimes \Lambda^{2}(\mathscr{G} / \mathscr{H})^{*} \longrightarrow 0,
$$

где

$$
\begin{aligned}
d_{1} p & =\widehat{y}(p) \partial_{y}+\widehat{z}(p) \partial_{z}=-x \frac{\partial p}{\partial x} \partial_{y}, \\
d_{2}\left(p_{1} \partial_{y}+p_{2} \partial_{z}\right) & =\left(\widehat{y}\left(p_{2}\right)-\widehat{z}\left(p_{1}\right)\right) \partial_{y} \wedge \partial_{z}=-x \frac{\partial p_{2}}{\partial x} \partial_{y} \wedge \partial_{z} .
\end{aligned}
$$

Таким образом, для $q=0$ получаем

$$
\begin{aligned}
E_{2}^{0,0} & =H^{0}(\mathscr{G} / \mathscr{H}, \mathbb{R}[x, z])=(\mathbb{R}[x, z])^{\mathscr{G} / \mathscr{H}}=\left\{p \mid x \frac{\partial p}{\partial x}=0\right\}=\mathbb{R}[z] \\
E_{2}^{1,0} & =H^{1}(\mathscr{G} / \mathscr{H}, \mathbb{R}[x, z])=\frac{Z^{1}}{B^{1}}=\frac{\left\{p_{1} \partial_{y}+p_{2} \partial_{z} \mid \partial p_{2} / \partial x=0\right\}}{\left\{-x \cdot \partial q / \partial x \cdot \partial_{y}\right\}} \\
& =\left\{p_{1}^{0}(z) \partial_{y}+p_{2}^{0}(z) \partial_{z}\right\}=(\mathscr{G} / \mathscr{H})^{*} \otimes \mathbb{R}[z] \\
E_{2}^{2,0} & =H^{2}(\mathscr{G} / \mathscr{H}, \mathbb{R}[x, z])=\frac{Z^{2}}{B^{2}}=\frac{\left\{p \partial_{y} \wedge \partial_{z}\right\}}{\left\{-x \cdot \partial p_{2} / \partial x \cdot \partial_{y} \wedge \partial_{z}\right\}} \\
& =\mathbb{R}[z] \partial_{y} \wedge \partial_{z}=\Lambda^{2}(\mathscr{G} / \mathscr{H})^{*} \otimes \mathbb{R}[z] .
\end{aligned}
$$

Для $q=1$ рассмотрим комплекс

$$
0 \longrightarrow \mathbb{R}[y, z] \partial_{x} \stackrel{d_{1}}{\longrightarrow} \mathbb{R}[y, z] \partial_{x} \otimes(\mathscr{G} / \mathscr{H})^{*} \stackrel{d_{2}}{\longrightarrow} \mathbb{R}[y, z] \partial_{x} \otimes \Lambda^{2}(\mathscr{G} / \mathscr{H})^{*} \longrightarrow 0,
$$

где для $p \in \mathbb{R}[y, z]$

$$
\begin{aligned}
d_{1}\left(p \partial_{x}\right) & =\widehat{y}\left(p \partial_{x}\right) \otimes \partial_{y}+\widehat{z}\left(p \partial_{x}\right) \otimes \partial_{z}=\left[-x \partial_{x}, p \partial_{x}\right] \otimes \partial_{y} \\
& =\left(p-x \frac{\partial p}{\partial x}\right) \partial_{x} \otimes \partial_{y}=p \partial_{x} \otimes \partial_{y}
\end{aligned}
$$

$$
\begin{aligned}
d_{2}\left(p_{1} \partial_{x} \otimes \partial_{y}+p_{2} \partial_{x} \otimes \partial_{z}\right) & =\widehat{y}\left(p_{2} \partial_{x}\right) \partial_{y} \wedge \partial_{z}=\left(p_{2}-x \frac{\partial p_{2}}{\partial x}\right) \partial_{x} \wedge \partial_{y} \wedge \partial_{z} \\
& =p_{2} \partial_{x} \wedge \partial_{y} \wedge \partial_{z} .
\end{aligned}
$$

Следовательно,

$$
\begin{aligned}
& E_{2}^{0,1}=H^{0}\left(\mathscr{G} / \mathscr{H}, \mathbb{R}[y, z] \partial_{x}\right)=\{p \mid p=0\} \otimes \partial_{x}=0, \\
& E_{2}^{1,1}=H^{1}\left(\mathscr{G} / \mathscr{H}, \mathbb{R}[y, z] \partial_{x}\right)=\frac{\left\{p_{1} \partial_{x} \wedge \partial_{y}+p_{2} \partial_{x} \wedge \partial_{z} \mid p_{2}=0\right\}}{\left\{q \partial_{x} \wedge \partial_{y}\right\}}=0, \\
& E_{2}^{2,1}=H^{2}\left(\mathscr{G} / \mathscr{H}, \mathbb{R}[y, z] \partial_{x}\right)=\frac{\left\{p \partial_{x} \wedge \partial_{y} \wedge \partial_{z}\right\}}{\left\{q \partial_{x} \wedge \partial_{y} \wedge \partial_{z}\right\}}=0 .
\end{aligned}
$$


В результате имеем следуюший вид члена $E_{2}^{p, q}$ спектральной последовательности:

\begin{tabular}{|c|c|c|c|c|}
\hline \multicolumn{4}{|c|}{$q_{\Lambda}$} & \multirow[t]{2}{*}{$E_{2}^{p, q}$} \\
\hline$q=1$ & 0 & 0 & 0 & \\
\hline$q=0$ & $\mathbb{R}[z]$ & $\mathbb{R}[z] \otimes(\mathscr{G} / \mathscr{H})^{*}$ & $\mathbb{R}[z] \otimes \Lambda^{2}(\mathscr{G} / \mathscr{H})^{*}$ & \\
\hline
\end{tabular}

или с учетом градуировки $S^{r} \mathscr{G}$

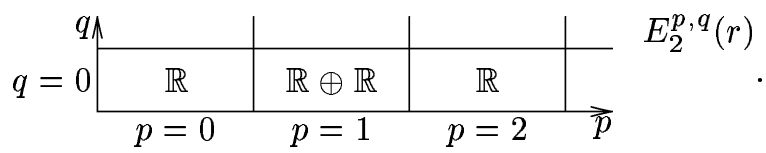

Из соображений размерности $d_{2}^{p, q}=d_{3}^{p, q}=\cdots=0$, следовательно, $E_{2}^{p, q}=E_{\infty}^{p, q}$.

Таким образом,

$$
H^{0}\left(\mathscr{G}, S^{r} \mathscr{G}\right)=\mathbb{R}, \quad H^{1}\left(\mathscr{G}, S^{r} \mathscr{G}\right)=\mathbb{R} \oplus \mathbb{R}, \quad H^{2}\left(\mathscr{G}, S^{r} \mathscr{G}\right)=\mathbb{R},
$$

а группа $H^{2}\left(\mathscr{G}, S^{*} \mathscr{G}\right)=\bigoplus_{r} H^{2}\left(\mathscr{G}, S^{r} \mathscr{G}\right)$ бесконечномерна.

Из приведенного описания следует, что препятствия для линеаризации пуассоновой структуры $\nabla$ с линейной частью $\nabla_{1}=x \partial_{x} \wedge \partial_{y}$, задающей алгебру Ли типа $\mathrm{A}$, лежат в $H^{2}\left(\mathscr{G}, S^{r} \mathscr{G}\right)$ и являются бивекторами вида $z^{r} \partial_{y} \wedge \partial_{z}, r \geqslant 2$.

Рассмотрим разность $\nabla-\nabla_{1} \in \mu^{2} \mathscr{D}_{2}$. Она является коциклом $\bmod \mu^{3} \mathscr{D}_{2}$. Пусть этот коцикл представлен бивектором $a_{2} z^{2} \partial_{y} \wedge \partial_{z}$. Рассмотрим $\nabla_{2}=x \partial_{x} \wedge \partial_{y}+a_{2} z^{2} \partial_{y} \wedge \partial_{z}$. Поскольку разность $\left[\nabla-\nabla_{2}\right]_{2}$ является 2-кограницей, сушествует локальный диффеоморфизм $\varphi_{2}$ такой, что $\left(\varphi_{2}\right)_{*}^{(2)}(\nabla)-\nabla_{2} \in \mu^{3} \mathscr{D}_{2}$.

Заметим, что любое бивекторное поле вида $\left(x \partial_{x}+p(z) \partial_{z}\right) \wedge \partial_{y}$ является пуассоновой структурой. Действительно, обозначим $a=x \partial_{x}+f(z) \partial_{z}, b=\partial_{y}$. Тогда

$$
\begin{aligned}
& \|[a \wedge b, a \wedge b]=-\| a \wedge b, a] \wedge b+a \wedge\|[a \wedge b, b]=-\| a, a \wedge b] \wedge b+a \wedge \| b, a \wedge b] \\
& \quad=\|a, a \rrbracket \wedge b \wedge b+a \wedge\|[a, b] \wedge b-a \wedge \| b, a] \wedge b-a \wedge a \wedge \llbracket b b, b]=2 a \wedge \llbracket[a, b] \wedge b=0 .
\end{aligned}
$$

Таким образом, $\nabla_{2}-$ пуассонова структура, поэтому $\left[\left(\varphi_{2}\right)_{*}^{(2)}(\nabla)-\nabla_{2}\right]_{3}-$ коцикл. Пусть он представлен бивектором $a_{3} z^{3} \partial_{y} \wedge \partial_{z}$. Рассмотрим пуассонову структуру $\nabla_{3}=x \partial_{x} \wedge$ $\partial_{y}+\left(a_{2} z^{2}+a_{3} z^{3}\right) \partial_{y} \wedge \partial_{z}$. Заметим, что $\left[\nabla-\nabla_{3}\right]_{3}$ является кограницей, поэтому существует локальный диффеоморфизм $\varphi_{3}$ такой, что $\left(\varphi_{3} \circ \varphi_{2}\right)_{*}^{(2)}(\nabla)-\nabla_{3} \in \mu^{4} \mathscr{D}_{2}$.

Продолжая этот процесс, мы получаем, что существует диффеоморфизм $\varphi$ такой, что $\varphi_{*}^{(2)}(\nabla)=\left(x \partial_{x} \wedge \partial_{y}+p(z) \partial_{y} \wedge \partial_{z}\right) \bmod \mu^{\infty} \mathscr{D}_{2}$, где $p(0)=p^{\prime}(0)=0$, т.е. $p(z)=a_{2} z^{2}+\cdots$. Векторное поле $p(z) \partial_{z}$ на прямой может быть приведено к нормальной форме $\pm Z^{r} \partial_{Z}$, которую для четного $r$ всегда можно брать со знаком “+”. Тем самым, доказана

ТЕОремА 1. Любая вырожденная пуассонова структура $\nabla$ в $\mathbb{R}^{3}$ с линейной частью, задающей алгебру Ли типа $\mathrm{A}$, формально әквивалентна пуассоновой структуре вида $\nabla_{r}=\left(x \partial_{x}+\varepsilon z^{r} \partial_{z}\right) \wedge \partial_{y}$, zде $r \geqslant 2 u$

$$
\varepsilon=\left\{\begin{aligned}
1 & \text { при четном } r \\
\pm 1 & \text { при нечетном } r \\
0 & \text { при } r=\infty \text { (формальная линеаризачия }) .
\end{aligned}\right.
$$


ЗАмЕчАниЕ. Рассмотрим следуюший подмодуль в модуле векторных полей:

$$
\left\{\nabla(d f) \mid f \in A=\mathscr{D}_{0}\right\} \subset \mathscr{D}_{1} .
$$

Траектории членов этого подмодуля определяют слоение размерности 2 на трехмерном многообразии (оно также задается поверхностями уровня функций Казимира), а слои называются пуассоновыми листами. Для пуассоновых структур $\nabla_{r}$ из теоремы 1 пуассоново слоение будет иметь особенность вдоль кривой $x=z=0$, и индуцированное слоение на трансверсали к этой кривой диффеоморфно слоению плоскости $\mathbb{R}(x, z)$ траекториями векторного поля $x \partial_{x}+\varepsilon z^{r} \partial_{z}$. Таким образом, для различных $r \in(\mathbb{N} \backslash\{1\}) \cup\{\infty\}$ пуассоновы структуры $\nabla_{r}$ неэквивалентны.

3.3. Алгебры типа В. Идеал $\mathscr{H} \subset \mathscr{G}$ порожден $z$, а факторалгебра $\mathscr{G} / \mathscr{H}$ порождена $x, y$. В данном случае $\widehat{x}=z \partial_{y}, \widehat{y}=-z \partial_{x}, \widehat{z}=0$.

В комплексе

$$
0 \longrightarrow \mathbb{R}[x, y, z] \stackrel{d_{1}}{\longrightarrow} \mathbb{R}[x, y, z] \otimes \mathscr{H}^{*} \longrightarrow 0
$$

дифференциал тривиален: $d_{1}(p)=\widehat{z}(p) \partial_{z}=0$. Поэтому

$$
H^{0}(\mathscr{H}, A)=\mathbb{R}[x, y, z], \quad H^{1}(\mathscr{H}, A)=\mathbb{R}[x, y, z] \partial_{z} .
$$

Для вычисления когомологий $\mathscr{G} / \mathscr{H}$ при $q=0$ рассмотрим комплекс

$$
0 \longrightarrow \mathbb{R}[x, y, z] \stackrel{d_{1}}{\longrightarrow} \mathbb{R}[x, y, z] \otimes(\mathscr{G} / \mathscr{H})^{*} \stackrel{d_{2}}{\longrightarrow} \mathbb{R}[x, y, z] \otimes \Lambda^{2}(\mathscr{G} / \mathscr{H})^{*} \longrightarrow 0
$$

где

$$
\begin{gathered}
d_{1}(p)=\widehat{x}(p) \partial_{x}+\widehat{y}(p) \partial_{y}=z \frac{\partial p}{\partial y} \partial_{x}-z \frac{\partial p}{\partial x} \partial_{y} \\
d_{2}\left(p_{1} \partial_{x}+p_{2} \partial_{y}\right)=\left(\widehat{x}\left(p_{2}\right)-\widehat{y}\left(p_{1}\right)\right) \partial_{x} \wedge \partial_{y}=z\left(\frac{\partial p_{2}}{\partial y}+\frac{\partial p_{1}}{\partial x}\right) \partial_{x} \wedge \partial_{y}
\end{gathered}
$$

Имеем

$$
Z^{1}=\left\{p_{1} \partial_{x}+p_{2} \partial_{y} \mid \frac{\partial p_{1}}{\partial x}+\frac{\partial p_{2}}{\partial y}=0\right\}, \quad B^{1}=\left\{z\left(\frac{\partial p}{\partial y} \partial_{x}-\frac{\partial p}{\partial x} \partial_{y}\right)\right\}
$$

Пусть

$$
p_{i}=\sum_{j=0}^{\infty} p_{i}^{j}(x, y) z^{j}
$$

Тогда

$$
\begin{aligned}
\frac{Z^{1}(\mathscr{G} / \mathscr{H}, \mathbb{R}[x, y, z])}{B^{1}(\mathscr{G} / \mathscr{H}, \mathbb{R}[x, y, z])}= & \bigoplus_{j=1}^{\infty}\left(\frac{\left\{p_{1}^{j}(x, y) \partial_{x}+p_{2}^{j}(x, y) \partial_{y} \mid \partial p_{1}^{j} / \partial x+\partial p_{2}^{j} / \partial y=0\right\}}{\left\{\partial p^{j-1}(x, y) / \partial y \cdot \partial_{x}-\partial p^{j-1}(x, y) / \partial x \cdot \partial_{y}\right\}} \otimes z^{j}\right) \\
& \oplus\left\{p_{1}^{0}(x, y) \partial_{x}+p_{2}^{0}(x, y) \partial_{y} \mid \frac{\partial p_{1}^{0}}{\partial x}+\frac{\partial p_{2}^{0}}{\partial y}=0\right\} .
\end{aligned}
$$

Легко видеть, что все слагаемые бесконечной суммы - нули, тем самым,

$$
\frac{Z^{1}}{B^{1}}=\left\{p_{1}^{0}(x, y) \partial_{x}+p_{2}^{0}(x, y) \partial_{y} \mid \frac{\partial p_{1}^{0}}{\partial x}+\frac{\partial p_{2}^{0}}{\partial y}=0\right\}
$$


Итак, первые когомологии указанного комплекса - бездивергентные поля на плоскости $\mathbb{R}(x, y)$ с формальньми коэффициентами. Эти поля совпадают с гамильтоновыми:

$$
\begin{aligned}
\mathscr{D}_{H} & =\left\{p_{1}^{0}(x, y) \partial_{x}+p_{2}^{0}(x, y) \partial_{y} \mid \frac{\partial p_{1}^{0}}{\partial x}+\frac{\partial p_{2}^{0}}{\partial y}=0\right\} \\
& =\left\{\frac{\partial p(x, y)}{\partial y} \partial_{x}-\frac{\partial p(x, y)}{\partial x} \partial_{y}\right\} \simeq \mathbb{R}[x, y] / \mathbb{R},
\end{aligned}
$$

где $\mathbb{R}[x, y]$ - формальные ряды, а $\mathbb{R}$ - свободные члены.

Заметим, что при переходе от гамильтоновых векторных полей к гамильтонианам градуировка поднимается на 1. Таким образом,

$$
\begin{gathered}
E_{2}^{0,0}=H^{0}(\mathscr{G} / \mathscr{H}, \mathbb{R}[x, y, z])=(\mathbb{R}[x, y, z])^{\mathscr{G} / \mathscr{H}}=\left\{p \mid \frac{\partial p}{\partial x}=\frac{\partial p}{\partial y}=0\right\}=\mathbb{R}[z] \\
E_{2}^{1,0}=H^{1}(\mathscr{G} / \mathscr{H}, \mathbb{R}[x, y, z])=\mathscr{D}_{H} \simeq \mathbb{R}[x, y] / \mathbb{R} .
\end{gathered}
$$

Вычислим вторые когомологии. Заметим, что когранищы $B^{2}$ порождены бивекторньми полями вида

$$
p \partial_{x} \wedge \partial_{y}=z\left(\frac{\partial p_{1}}{\partial x}+\frac{\partial p_{2}}{\partial y}\right) \partial_{x} \wedge \partial_{y} .
$$

Очевидно, что любое (формальное) бивекторное поле на $\mathbb{R}(x, y)$ представимо в таком виде, при условии, что оно кратно $z$. Таким образом,

$$
E_{2}^{2,0}=H^{2}(\mathscr{G} / \mathscr{H}, \mathbb{R}[x, y, z])=\frac{Z^{2}}{B^{2}}=\mathbb{R}[x, y] \partial_{x} \wedge \partial_{y}
$$

Для вычисления членов спектральной последовательности при $q=1$ имеем следующий комплекс:

$$
0 \longrightarrow \mathbb{R}[x, y, z] \partial_{z} \stackrel{d_{1}}{\rightarrow} \mathbb{R}[x, y, z] \partial_{z} \otimes(\mathscr{G} / \mathscr{H})^{*} \stackrel{d_{2}}{\longrightarrow} \mathbb{R}[x, y, z] \partial_{z} \otimes \Lambda^{2}(\mathscr{G} / \mathscr{H})^{*} \longrightarrow 0,
$$

где

$$
\begin{gathered}
d_{1}\left(p \partial_{z}\right)=\widehat{x}(p) \partial_{z} \otimes \partial_{x}+\widehat{y}(p) \partial_{z} \otimes \partial_{y}=z \partial_{z} \wedge\left(\frac{\partial p}{\partial y} \partial_{x}-\frac{\partial p}{\partial x} \partial_{y}\right) \\
d_{2}\left(p_{1} \partial_{z} \wedge \partial_{x}+p_{2} \partial_{z} \wedge \partial_{y}\right)=z\left(\frac{\partial p_{1}}{\partial x}+\frac{\partial p_{2}}{\partial y}\right) \partial_{z} \wedge \partial_{x} \wedge \partial_{y}
\end{gathered}
$$

Поскольку этот комплекс отличается от предыдущего сомножителем $\partial_{z}$, коммутирующим с дифференциалами, то

$$
\begin{gathered}
E_{2}^{0,1}=H^{0}\left(\mathscr{G} / \mathscr{H}, \mathbb{R}[x, y, z] \partial_{z}\right)=\mathbb{R}[z] \partial_{z} \\
E_{2}^{1,1}=H^{1}\left(\mathscr{G} / \mathscr{H}, \mathbb{R}[x, y, z] \partial_{z}\right)=\mathscr{D}_{H} \wedge \partial_{z} \simeq(\mathbb{R}[x, y] / \mathbb{R})^{1 \uparrow} \wedge \partial_{z} \\
E_{2}^{2,1}=H^{2}\left(\mathscr{G} / \mathscr{H}, \mathbb{R}[x, y, z] \partial_{z}\right)=\mathbb{R}[x, y] \partial_{x} \wedge \partial_{y} \wedge \partial_{z}
\end{gathered}
$$

где через $(\cdot)^{1 \uparrow}$ обозначен оператор повьшения градуировки на 1 . В результате спектральная последовательность имеет следующий вид:

\begin{tabular}{rl|c|c|c}
$q_{1}$ & & & \\
\cline { 2 - 5 } & $S^{*} \mathscr{H}$ & $\left(S^{*}(\mathscr{G} / \mathscr{H}) / \mathbb{R}\right)^{1 \uparrow}$ & $S^{*}(\mathscr{G} / \mathscr{H})$ & \\
\cline { 2 - 5 } & $S^{*} \mathscr{H}$ & $\left(S^{*}(\mathscr{G} / \mathscr{H}) / \mathbb{R}\right)^{1 \uparrow}$ & $S^{*}(\mathscr{G} / \mathscr{H})$ & \\
& \multicolumn{2}{c}{$p=1$} & $p=2$ & $\bar{p}$
\end{tabular},


или для $r$-компоненты градуировки $A=S^{*} \mathscr{G}=\bigoplus_{r=0}^{\infty} S^{r} \mathscr{G}$ :

\begin{tabular}{|c|c|c|c|c|}
\hline \multicolumn{2}{|c|}{$q_{\Lambda}$} & & & \multirow[t]{2}{*}{$E_{2}^{p, q}(r)$} \\
\hline$q=1$ & $S^{r} \mathscr{H}$ & $S^{r+1}(\mathscr{G} / \mathscr{H})$ & $S^{r}(\mathscr{G} / \mathscr{H})$ & \\
\hline$q=0$ & $S^{r} \mathscr{H}$ & $S^{r+1}(\mathscr{G} / \mathscr{H})$ & $S^{r}(\mathscr{G} / \mathscr{H})$ & \\
\hline & $p=0$ & $p=1$ & $p=2$ & \\
\hline
\end{tabular}

В этой спектральной последовательности имеется лишь один нетривиальный дифференциал - отображение трансгрессии $d_{2}^{0,1}: E_{2}^{0,1} \rightarrow E_{2}^{2,0}$. Вычислим это отображение. Пусть $p(z) \partial_{z}$ - представитель элемента из $E_{2}^{0,1}$. Тогда по определению $d_{2}^{0,1}$ имеем

$$
d_{2}^{0,1}\left[p(z) \partial_{z}\right]_{2}^{0,1}=\left[d\left(p(z) \partial_{z}\right)\right]_{2}^{2,0},
$$

где через $[\cdot]_{2}^{p, q}$ обозначены классы соответствующих элементов в $E_{2}^{p, q}$.

Имеем

$$
\begin{aligned}
d\left(p(z) \partial_{z}\right) & =\widehat{x}\left(p(z) \partial_{z}\right) \wedge \partial_{x}+\widehat{y}\left(p(z) \partial_{z}\right) \wedge \partial_{y} \\
& =\left(z \frac{\partial p(z)}{\partial y} \partial_{z}+p(z)\left[z \partial_{y}, \partial_{z}\right]\right) \wedge \partial_{x}+\left(-z \frac{\partial p(z)}{\partial x} \partial_{z}+p(z)\left[-z \partial_{x}, \partial_{z}\right]\right) \wedge \partial_{y} \\
& =\left(-p(z) \partial_{y}\right) \wedge \partial_{x}+\left(p(z) \partial_{x}\right) \wedge \partial_{y}=2 p(z) \partial_{x} \wedge \partial_{y} .
\end{aligned}
$$

После факторизации в силу (4) получаем

$$
d_{2}^{0,1}\left[p(z) \partial_{z}\right]_{2}^{0,1}=\left[2 p(0) \partial_{x} \wedge \partial_{y}\right]_{2}^{2,0}
$$

т.е.

$$
\operatorname{Ker} d_{2}^{0,1}=(\mathbb{R}[z] / \mathbb{R}) \otimes \partial_{z} \simeq(\mathbb{R}[z])^{1 \downarrow}, \quad \text { Coker } d_{2}^{2,0}=(\mathbb{R}[x, y] / \mathbb{R}) \otimes\left(\partial_{x} \wedge \partial_{y}\right) .
$$

Таким образом, имеем следующее представление члена $E_{3}^{p, q}$ :

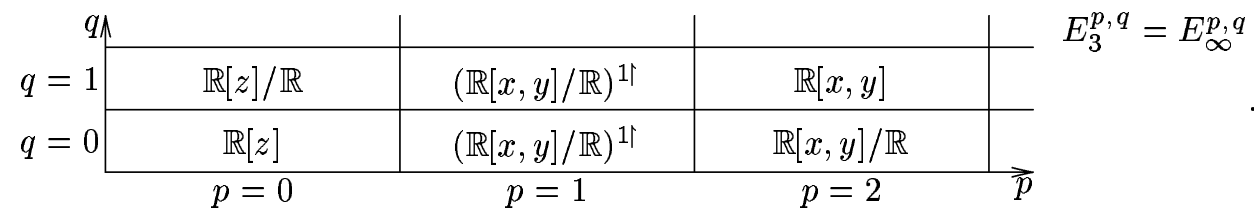

Следовательно, группа $H^{2}\left(\mathscr{G}, S^{*} \mathscr{G}\right)=\bigoplus_{r} H^{2}\left(\mathscr{G}, S^{r} \mathscr{G}\right)$ бесконечномерна.

Рассмотрим пуассонову структуру $\nabla$ в $\mathbb{R}^{3}$ с линейной частью $\nabla_{1}=z \partial_{x} \wedge \partial_{y}$, задающей алгебру Ли типа В.

Как следует из вычисления когомологий $H^{2}\left(\mathscr{G}, S^{r} \mathscr{G}\right)$, препятствия к линеаризации $\nabla$ могут быть двух видов: бивекторы $p(x, y) \partial_{x} \wedge \partial_{y}, p \in \mu^{2}$ (из $E_{\infty}^{2,0}$ ), а также бивекторы вида $X_{H} \wedge \partial_{z}$, где

$$
X_{H}=\frac{\partial H}{\partial x} \partial_{y}-\frac{\partial H}{\partial y} \partial_{x}
$$

- гамильтоново векторное поле на плоскости $\mathbb{R}(x, y), H \in \mu^{3}$ (из $E_{\infty}^{1,1}$ ).

Таким образом (теорема A), пуассонова структура $\nabla$ с точностью до членов третьего порядка малости приводится к виду

$$
\left(z+p_{2}(x, y)\right) \partial_{x} \wedge \partial_{y}+X_{H} \wedge \partial_{y},
$$

где $p_{2}(x, y)$ - квадратичный, а $H(x, y)$ - кубический многочлены. С помощью преобразования из $\mathrm{sl}(2, \mathbb{R})$ квадрика $p_{2}$ приводится к главным осям. При этом сохраняется максимальньй бивектор $\partial_{x} \wedge \partial_{y}$. Так как $\mathrm{sl}(2, \mathbb{R})=\operatorname{sp}(2, \mathbb{R})$, такие преобразования переводят гамильтоновы векторные поля в гамильтоновы. Итак, доказана 
Теорема 2. Для любой пуассоновой структуры $\nabla$ в $\mathbb{R}^{3}$ с линейной частью $\nabla_{1}=z \partial_{x} \wedge \partial_{y}$, задающей алгебру Ли типа $\mathrm{B}$, существует диффеоморфизм $\varphi$ такой, что $\varphi^{*}=\operatorname{id} \bmod \mu^{2} u$

$$
(\varphi)_{*}^{(2)}(\nabla)=\left(\left(z+\lambda_{1} x^{2}+\lambda_{2} y^{2}\right) \partial_{x} \wedge \partial_{y}+X_{H} \wedge \partial_{z}\right) \bmod \mu^{3} \mathscr{D}_{2},
$$

где $X_{H}$ - гамильтоново векторное поле на плоскости $\mathbb{R}(x, y)$, а $H$ - однородный кубический полином.

3.4. Алгебры типа С. Рассмотрим теперь случай двумерного коммутанта $\mathscr{H} \subset \mathscr{G}$. Пусть идеал $\mathscr{H}$ порожден $x, y$, а $\mathscr{G} / \mathscr{H}$ порождена $z$, и $[x, y]=0,[z, x]=f=a_{11} x+a_{12} y$, $[z, y]=g=a_{21} x+a_{22} y$. В этом случае $\widehat{x}=-f \partial_{z}, \widehat{y}=-g \partial_{z}, \widehat{z}=f \partial_{x}+g \partial_{y}$.

Начнем с вычисления когомологий $\mathscr{H}$ с коэффициентами в $A=S^{*} \mathscr{G}$. Из комплекса

$$
0 \longrightarrow \mathbb{R}[x, y, z] \stackrel{d_{1}}{\longrightarrow} \mathbb{R}[x, y, z] \otimes \mathscr{H}^{*} \stackrel{d_{2}}{\longrightarrow} \mathbb{R}[x, y, z] \otimes \Lambda^{2} \mathscr{H}^{*} \longrightarrow 0
$$

где

$$
\begin{aligned}
d_{1} p & =\widehat{x}(p) \partial_{x}+\widehat{y}(p) \partial_{y}=-f \frac{\partial p}{\partial z} \partial_{x}-g \frac{\partial p}{\partial z} \partial_{y} \\
d_{2}\left(p_{1} \partial_{x}+p_{2} \partial_{y}\right) & =\left(\widehat{x}\left(p_{2}\right)-\widehat{y}\left(p_{1}\right)\right) \partial_{x} \wedge \partial_{y}=\left(g \frac{\partial p_{1}}{\partial z}-f \frac{\partial p_{2}}{\partial z}\right) \partial_{x} \wedge \partial_{y}
\end{aligned}
$$

получаем

$$
\begin{gathered}
H^{0}(\mathscr{H}, A)=A^{\mathscr{H}}=\left\{p \mid \frac{\partial p}{\partial z}=0\right\}=\mathbb{R}[x, y], \\
H^{1}(\mathscr{H}, A)=\frac{Z^{1}}{B^{1}}=\frac{\left\{p_{1} \partial_{x}+p_{2} \partial_{y} \mid g \cdot \partial p_{1} / \partial z-f \cdot \partial p_{2} / \partial z=0\right\}}{\left\{f \cdot \partial p / \partial z \cdot \partial_{x}+g \cdot \partial p / \partial z \cdot \partial_{y}\right\}} .
\end{gathered}
$$

Нетрудно проверить, что данное факторпространство совпадает с $\operatorname{Vect}(x, y) /\langle\mathbb{R}[x, y] \widehat{z}\rangle$, т.е.

$$
H^{1}(\mathscr{H}, A)=\frac{\operatorname{Vect}(x, y)}{\langle\mathbb{R}[x, y] \widehat{z}\rangle},
$$

где $\operatorname{Vect}(x, y)$ - модуль всех формальных векторных полей на плоскости $\mathbb{R}(x, y)$.

Далее имеем

$$
H^{2}(\mathscr{H}, A)=\frac{\left\{p \partial_{x} \wedge \partial_{y}\right\}}{\left\{\left(g \cdot \partial p_{1} / \partial z-f \cdot \partial p_{2} / \partial z\right) \partial_{x} \wedge \partial_{y}\right\}}=\mathbb{R}[z] \partial_{x} \wedge \partial_{y}=\mathbb{R}[z] \otimes \Lambda^{2} \mathscr{H}^{*}
$$

Для вычисления $E_{2}^{0,0}$ и $E_{2}^{1,0}$ имеем комплекс

$$
0 \longrightarrow \mathbb{R}[x, y] \stackrel{d}{\longrightarrow} \mathbb{R}[x, y] \otimes(\mathscr{G} / \mathscr{H})^{*} \longrightarrow 0,
$$

где

$$
d p=\widehat{z}(p) \partial_{z}=\left(f \frac{\partial p}{\partial x}+g \frac{\partial p}{\partial y}\right) \partial_{z} .
$$


Поэтому $E_{2}^{0,0}=H^{0}(\mathscr{G} / \mathscr{H}, \mathbb{R}[x, y])=I$ - алгебра (формальньх) первых интегралов векторного поля $\widehat{z}$ на плоскости $\mathbb{R}(x, y)$, а

$$
E_{2}^{1,0}=H^{1}(\mathscr{G} / \mathscr{H}, \mathbb{R}[x, y])=\mathbb{R}[x, y] / \widehat{z}(\mathbb{R}[x, y]) .
$$

Соответственно из комплекса

$$
0 \longrightarrow H^{1}(\mathscr{H}, A) \longrightarrow H^{1}(\mathscr{H}, A) \otimes(\mathscr{G} / \mathscr{H})^{*} \longrightarrow 0
$$

мы получаем, что

$$
E_{2}^{0,1}=\left(H^{1}(\mathscr{H}, A)\right)^{\widehat{z}}, \quad E_{2}^{1,1}=H^{1}\left(\mathscr{G} / \mathscr{H}, H^{1}(\mathscr{H}, A)\right) \simeq \frac{H^{1}(\mathscr{H}, A)}{\widehat{z}\left(H^{1}(\mathscr{H}, A)\right)} .
$$

Для вычисления этих когомологий воспользуемся описанием $H^{1}(\mathscr{H}, A)$ и введем отображение $\Phi: \operatorname{Vect}(x, y) \rightarrow \Lambda^{2} \operatorname{Vect}(x, y)$ из пространства векторных полей на плоскости $\mathbb{R}(x, y)$ в пространство бивекторных, определенное внешним умножением на $\widehat{z}: v \mapsto v \wedge \widehat{z}$.

Заметим, что $\operatorname{Ker}(\Phi)=\langle\widehat{z}\rangle=\{p(x, y) \widehat{z}\}$. Следовательно, в силу (5) образ $\operatorname{Im} \Phi$ отображения $\Phi$ изоморфен групе $H^{1}(\mathscr{H}, A)$. Кроме того, при изоморфизме $\Phi$ градуировка увеличивается на 1 .

Найдем образ дифференцирования $\widehat{z}$ при изоморфизме $\widetilde{\Phi}: H^{1}(\mathscr{H}, A) \rightarrow \operatorname{Im} \Phi$. Имеем

$$
\Phi(\widehat{z}(v))=\widehat{z}(v) \wedge \widehat{z}=L_{\widehat{z}}(v \wedge \widehat{z})=L_{\widehat{z}} \Phi(v),
$$

т.е. $\widehat{z}$ действует как производная Ли $L_{\widehat{z}}$.

Имеем

$$
\widehat{z}\left(p(x, y) \partial_{x} \wedge \partial_{y}\right)=(\widehat{z}(p)-p \operatorname{div} \widehat{z}) \partial_{x} \wedge \partial_{y}
$$

где $\operatorname{div} \widehat{z}=\operatorname{tr}\left(a_{i j}\right)=a_{11}+a_{22}$.

Итак, мы имеем индуцированное действие векторного поля $\widehat{z}$ в $H^{1}(\mathscr{H}, A)$, изоморфной $S^{*}(x, y)$ :

$$
D_{\widehat{z}}^{r+1}: S^{r+1}(x, y) \rightarrow S^{r+1}(x, y)
$$

где $D_{\widehat{z}}^{r+1}$ есть $(r+1)$-я компонента оператора (6).

Пусть $\lambda_{1}, \lambda_{2}$ - собственные числа оператора $S^{1}(x, y) \rightarrow S^{1}(x, y)$, т.е. собственные числа матрицы $\left(a_{i j}\right)$. Тогда собственньми числами оператора $D_{\widehat{z}}^{r+1}$ на $S^{r+1}$ являются

$$
\left\{m_{1} \lambda_{1}+m_{2} \lambda_{2}-d \mid m_{1}+m_{2}=r+1, m_{1}, m_{2} \geqslant 0, r \geqslant 2\right\},
$$

где $d=\operatorname{div} \widehat{z}=\lambda_{1}+\lambda_{2}$. Таким образом, спектр оператора $D_{\widehat{z}}^{r+1}$ совпадает с множеством

$$
\Pi^{r+1}\left(\lambda_{1}, \lambda_{2}\right)=\left\{\left(m_{1}-1\right) \lambda_{1}+\left(m_{2}-1\right) \lambda_{2} \mid m_{1}+m_{2}=r+1, m_{1}, m_{2} \geqslant 0\right\}, \quad r \geqslant 2 .
$$

Таким образом, если в наборе чисел $\Pi^{r+1}\left(\lambda_{1}, \lambda_{2}\right)$ отсутствуют нули, то $D_{\widehat{z}}^{r+1}$, а значит и $\widehat{z}: H^{1}\left(\mathscr{H}, S^{r} \mathscr{G}\right) \rightarrow H^{1}\left(\mathscr{H}, S^{r} \mathscr{G}\right)$, является изоморфизмом.

Заметим, что образ $\operatorname{Im} \Phi$ совпадает с множеством всех бивекторов, обращающихся в 0 в точке $(x, y)=(0,0)$, т.е. $\operatorname{Im} \Phi=S^{*} \mathscr{H} / \mathbb{R} \otimes \Lambda^{2} \mathscr{H}$, где $\mathbb{R}$ - свободные члены. Кроме того, $\widehat{z}$ сохраняет градуировку, и $\left(S^{*} \mathscr{H} \otimes \Lambda^{2} \mathscr{H}\right) / \operatorname{Im} \Phi=\mathbb{R}$ отвечает нулевому уровню градуировки. 
Итак, в случае отсутствия нулей в $\Pi^{r+1}\left(\lambda_{1}, \lambda_{2}\right)$ имеем

$$
E_{2}^{0,1}=\operatorname{Ker} \mathscr{D} \widehat{z}=0, \quad E_{2}^{1,1}=H^{1}\left(\mathscr{G} / \mathscr{H}, H^{1}(\mathscr{H}, A)\right)=\frac{\operatorname{Im} \Phi}{\widehat{z}(\operatorname{Im} \Phi)}=\frac{\operatorname{Im} \Phi}{\operatorname{Im} \Phi}=0 .
$$

Если же в $\Pi^{r+1}\left(\lambda_{1}, \lambda_{2}\right)$ имеются нули, то члены $E_{2}^{0,1}(r)$ и $E_{2}^{1,1}(r)$ ненулевые.

Наконец, вычислим верхнюю строчку спектральной последовательности:

$$
\begin{gathered}
E_{2}^{0,2}=H^{0}\left(\mathscr{G} / \mathscr{H}, H^{2}(\mathscr{H}, A)\right)=H^{0}\left(\mathscr{G} / \mathscr{H}, \mathbb{R}[z] \partial_{x} \wedge \partial_{y}\right)=\left(\mathbb{R}[z] \partial_{x} \wedge \partial_{y}\right)^{\mathscr{G} / \mathscr{H}}, \\
E_{2}^{1,2}=H^{1}\left(\mathscr{G} / \mathscr{H}, H^{2}(\mathscr{H}, A)\right)=\frac{H^{2}(\mathscr{H}, A)}{\widehat{z}\left(H^{2}(\mathscr{H}, A)\right)} .
\end{gathered}
$$

Найдем действие $\widehat{z}$ в $H^{2}(\mathscr{H}, A)$ :

$\widehat{z}\left(p(z) \partial_{x} \wedge \partial_{y}\right)=p(z)\left(\left[\widehat{z}, \partial_{x}\right] \wedge \partial_{y}+\partial_{x} \wedge\left[\widehat{z}, \partial_{y}\right]\right)=-(\operatorname{div} \widehat{z}) p(z) \partial_{x} \wedge \partial_{y}=-d \cdot p(z) \partial_{x} \wedge \partial_{y}$.

Таким образом, если $d=\operatorname{div} \widehat{z}=\lambda_{1}+\lambda_{2} \neq 0$, то дифференциал следующего комплекса является изоморфизмом:

$$
0 \longrightarrow H^{2}(\mathscr{H}, A) \stackrel{\text { iso }}{\longrightarrow} H^{2}(\mathscr{H}, A) \otimes(\mathscr{G} / \mathscr{H})^{*} \longrightarrow 0,
$$

и мы имеем $E_{2}^{1,2}=E_{2}^{0,2}=0$.

Итак, спектральная последовательность $E_{2}^{p, q}$ имеет вид

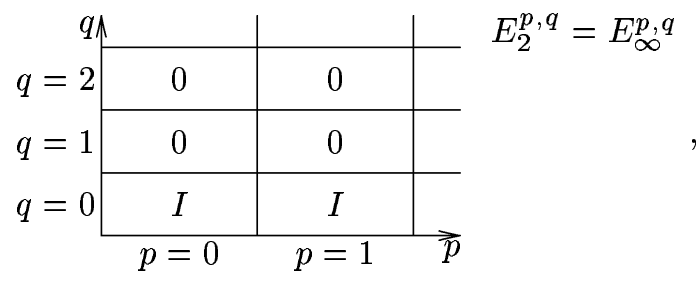

если $d \neq 0$ и $\Pi^{r+1}\left(\lambda_{1}, \lambda_{2}\right)$ не содержит нулей.

В качестве следствия мы получаем

ПреДЛОЖенИЕ. Если собственные числа $\lambda_{1}, \lambda_{2}$ оператора $\widehat{z}: \mathscr{H} \rightarrow \mathscr{H}$ таковы, umo $\lambda_{1}+\lambda_{2} \neq 0 u\{0\} \notin \Pi^{r+1}\left(\lambda_{1}, \lambda_{2}\right)$, mo $H^{2}\left(\mathscr{G}, S^{r} \mathscr{G}\right)=0$.

Теорема 3. Пусть $\nabla$ - вырожденная пуассонова структура в $\mathbb{R}^{3}$ с линейной частью типа С и $\lambda_{1}, \lambda_{2}-$ собственные значения матриць $\left(a_{i j}\right)$.

Тогда $\nabla$ формально әквивалентна линейной пуассоновой структуре

$$
\left(a_{11} x+a_{12} y\right) \partial_{z} \wedge \partial_{x}+\left(a_{21} x+a_{22} y\right) \partial_{z} \wedge \partial_{y},
$$

если выполнено одно из следующих условий:

1) $\lambda_{1}, \lambda_{2} \in \mathbb{R}, \lambda_{1} \lambda_{2}>0 u \lambda_{1} / \lambda_{2} \notin \mathbb{N} \backslash\{1\}, \lambda_{2} / \lambda_{1} \notin \mathbb{N} \backslash\{1\}$;

2) $\lambda_{1}, \lambda_{2} \in \mathbb{R}, \lambda_{1} \lambda_{2}<0 u \lambda_{1} / \lambda_{2} \notin \mathbb{Q}$;

3) $\lambda_{1}=\bar{\lambda}_{2} \notin \mathbb{R}, \operatorname{Re} \lambda_{1} \neq 0$. 
ЗАмЕчАниЕ. Две матрищы $\left(a_{i j}\right)$ и $\left(b_{i j}\right)$ в классификации Бьянки определяют изоморфные алгебры Ли тогда и только тогда, когда существуют обратимая матрица $\left(c_{i j}\right)$ и число $l \neq 0$ такие, что $l \cdot\left(a_{i j}\right)=\left(c_{i j}\right)\left(b_{i j}\right)\left(c_{i j}\right)^{-1}$.

В силу этого замечания мы можем упростить формулировку теоремы 3 . Рассмотрим матрицу $\left(a_{i j}\right)$. Пусть матрица $\left(c_{i j}\right)$ такова, что $\left(c_{i j}\right)\left(a_{i j}\right)\left(c_{i j}\right)^{-1}$ имеет каноническую форму, т.е. является матрицей одного из следующих типов.

1. Диагональная матрища: $\left(\begin{array}{cc}\lambda_{1} & 0 \\ 0 & \lambda_{2}\end{array}\right)$. Умножением на скалярные матрицы может быть приведена к виду $\left(\begin{array}{ll}1 & 0 \\ 0 & \nu\end{array}\right)$.

2. Жорданова клетка: $\left(\begin{array}{ll}\lambda & 1 \\ 0 & \lambda\end{array}\right)$. Умножением на скалярные матрицы и линейными преобразованиями может быть приведена к виду $\left(\begin{array}{ll}1 & \beta \\ 0 & 1\end{array}\right)$, где $\beta \neq 0$.

3. Комплексная матрища $c$ ненулевой действительной частью приводится к виду $\left(\begin{array}{cc}1 & \varkappa \\ -\varkappa & 1\end{array}\right)$, где $\varkappa \in \mathbb{R}$.

Теорема 4. В условиях теоремы 3 пуассонова структура $\nabla$ формально әквивалентна одной из следующих линейных структур:

1) $\partial_{z} \wedge\left(x \partial_{x}+\nu y \partial_{y}\right)$, где $\nu \notin \mathbb{Q}_{-} \cup\{1 / n \mid n \in \mathbb{N} \backslash\{1\}\} \cup(\mathbb{N} \backslash\{1\})$;

2) $\partial_{z} \wedge\left((x+\beta y) \partial_{x}+y \partial_{y}\right)$

3) $\partial_{z} \wedge\left((x+\varkappa y) \partial_{x}+(y-\varkappa x) \partial_{y}\right)$.

Автор благодарен рецензенту за указание ссылки [3] и другие полезные замечания.

\section{СПИСОК ЦИТИРОВАННОЙ ЛИТЕРАТУРЫ}

[1] Weinstein A. The local structure of Poisson manifolds // J. Differential Geom. 1983. V. 18. P. 523-557.

[2] Conn J.F. Normal forms for smooth Poisson structures // Ann. of Math. (2). 1985. V. 121. P. 565-593.

[3] Dufour J.-P. Linéarisation de certaines structures de Poisson // J. Differential Geom. 1990. V. 32. P. 415-428.

[4] Лычагина О.В. Классификация пуассоновых структур // Докл. РАН. 1996. Т. 350. №3. C. $304-307$.

[5] Лычагина О. В. Нормальные формы вырожденньх пуассоновых структур // Матем. заметки. 1997. Т. 61. № 2. С. 220-235.

[6] Карасёв М. В., Маслов В. П. Нелинейные скобки Пуассона. Геометрия и квантование. М.: Наука, 1991.

[7] Дубровин Б. А., Новиков С. П., Фоменко А. Т. Современная геометрия. М.: Наука, 1979.

[8] Hochschild G., Serre J.-P. Cohomology of Lie Algebras // Ann. of Math. 1953. V. 57. P. 591-603.

[9] Арнольд В.И. Математические методы классической механики. М.: Наука, 1984.

Научно-исследовательское предприятие "ОСТ", г. Москва

Поступило

E-mail: lychagin@glasnet.ru

18.09 .96

Исправленный вариант 10.11.97 